\title{
Modified Anterior Craniofacial Osteotomy Using Partial Nasal Bone Division and Reconstruction in Frontoethmoidal Sinus Meningioma
}

Eon Ju Park, Hong II Kim, Jin Hyung Park, Hyung Suk Yi

Department of Plastic and Reconstructive Surgery, Kosin University College of Medicine, Busan, Korea

No potential conflict of interest relevant to this article was reported.

\begin{abstract}
Typical transcranial approaches are insufficient for adequate visualization and resection of skull base tumors. Different approaches with multiple modifications have been attempted. Here, we describe a new approach for a lesion that is central and hard to treat by conventional craniotomy and successful reconstruction with calvarial bone graft and titanium mesh plate. A 69-year-old female patient presented with recurrent meningioma. The tumor had invaded the frontal lobe, right supraorbital rim, and ethmoidal bone. We performed a modified anterior craniofacial approach that fully exposed the tumor and invaded bone. In consideration of the patient's age and cosmetic result, the tumor and invaded bone was resected and the defect area was reconstructed with titanium mesh and calvarial bone graft. At 6 months postoperative the patient had no complications and was satisfied with the esthetic result. We report this case to demonstrate the successful approach and reconstruction using this technique.
\end{abstract}

Keywords: Meningioma / Osteotomy / Autograft

\section{INTRODUCTION}

Craniofacial surgeons have developed several approaches to widen the operation view and minimize facial deformity after reconstruction during surgery. Since the first report of orbitozygomatic craniotomy in 1984, this technique has been widely adopted due to the advantage of deep lesion exposure with shorter distance and minimal brain retraction [1]. However, in many cases typical transcranial approaches are insufficient for adequate visualization and resection of skull base tumors [2]. Especially, surgical access to a central intracranial tumor is limited due to the centrality of lesions surrounded by neurovascular structures. Different approaches with multiple modifications are available for the treatment of midline cranial base lesions [3]; however, conventional

Correspondence: Hyung Suk Yi

Department of Plastic and Reconstructive Surgery, Kosin University College of Medicine, 262 Gamcheon-ro, Seo-gu, Busan 49267, Korea

E-mail: sencha21@naver.com

Received February 16, 2017 / Revised May 10, 2017 / Accepted May 10, 2017 approaches are associated with a limited operation field and unfavorable postoperative cranio-facial outcomes.

In the present case, we designed a new approach for a lesion that was central and hard to treat by conventional craniotomy. We performed a modified anterior craniofacial approach and reconstruction with calvarial bone graft and titanium mesh plate. We report successful approach and reconstruction using this technique.

\section{CASE REPORT}

A 69-year-old female patient presented with recurrent meningioma on the frontal lobe area. The patient underwent tumor resection three times previously. Her chief complains were headache and diplopia. Neuroimaging with magnetic resonance imaging (MRI) revealed a $5.5 \times 2.8 \mathrm{~cm}$ sized ethmoid-orbital meningioma and hyperostotic lesion in supraorbital rim and frontal and ethmoid sinuses (Fig. 1). 


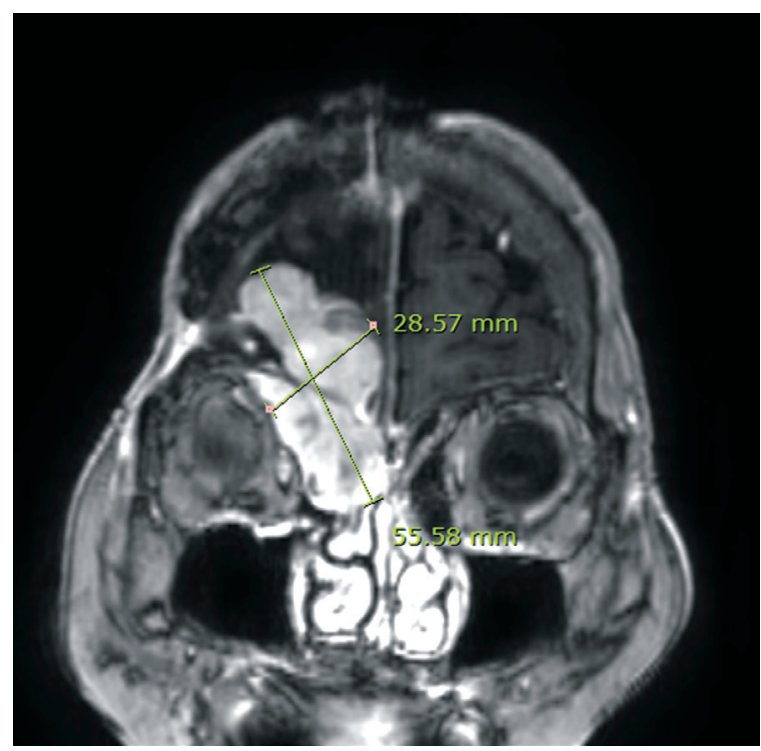

Fig. 1. Coronal view of post-contrast T1 magnetic resonance imaging scan demonstrated an approximately $5.5 \times 2.8 \mathrm{~cm}$ sized heterogeneous enhancing mass (meningioma) involving the right frontal convexity and extending into right supramedial extraconal space and frontal and ethmoidal sinuses.

A
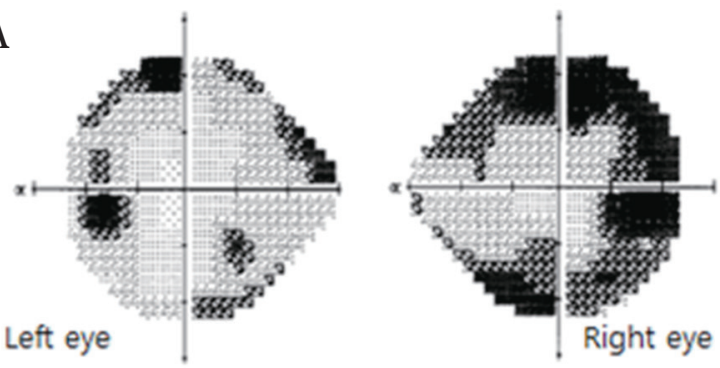

B
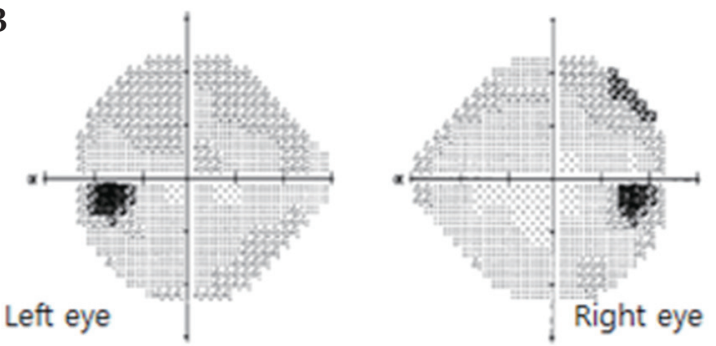

Fig. 2. (A) Preoperative visual field. Moderate peripheral field loss on right eye and mild peripheral field loss on left eye were observed. (B) Postoperative visual field. Peripheral field loss on both eyes had disappeared.

Both preoperative visual acuity test scales were 0.8 Log minimum angle of resolution (MAR). Visual field test showed dominant peripheral field loss in the right eye and mild peripheral field
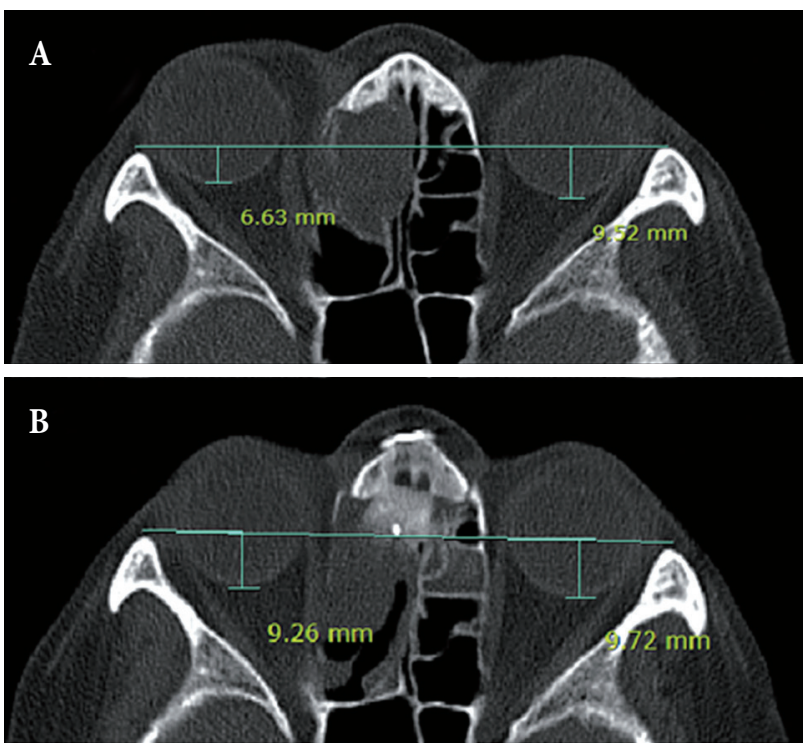

Fig. 3. Proptosis is evaluated by the perpendicular distance between the interzygomatic line and the posterior margin of the globe at the mid-globe sections. (A) Preoperative axial computed tomography (CT) of the orbit in soft tissue windows. (B) Postoperative axial CT of the orbit in soft tissue windows. There was a $2.89-\mathrm{mm}$ difference between the eyes before surgery and a $0.46-\mathrm{mm}$ difference after surgery.

loss in the left eye (Fig. 2A). Visual field loss resulted from compression of the optic artery and nerve by the menigioma. The right eye exhibited extraocular muscle (EOM) limitation with upper gaze and proptosis (Fig. 3A). Proptosis is evaluated by the perpendicular distance between the interzygomatic line and the posterior margin of the globe at the mid-globe sections on computed tomography (CT) axial view [4]. There was a 2.89-mm difference between the two eyes. With the patient under general anesthesia, the plastic surgeon (PS) made a bicoronal incision, elevated the frontal flap, and carefully released the supraorbital nerves. The field was fully exposed from frontal bone to nasal bone. Anterior and posterior frontal wall and sinus showed hyperostotic lesions indicating invasion of the meningioma. These lesions were resected via craniotomy by a neurosurgeon (NS). Although the tumor had invaded the anterior wall of the frontal bone, it was not completely removed because of patient age and the potential for postoperative complications. After consultation with the NS, only the right orbital roof and the posterior wall of the frontal sinus were removed. The outer cortex was reused. Mucous tissues of the frontal sinus were obliterated using electrocautery. The PS de- 
signed a modified osteotomy approach for this case. A vertical line was designed $1 \mathrm{~cm}$ lateral from the supraorbital notch on the right side of supraorbital rim and $1 \mathrm{~cm}$ medial from the supraorbital notch on the left side with consideration of tumor location and size on preoperative MRI. A transverse line was designed at the midpoint of the nasal bone. Full-thickness osteotomy was performed with a reciprocating saw at the vertical line of the supraorbital rim. Partial osteotomy at the transverse line of nasal bone was performed with an oscillating saw. Osteotomy was completed with a 6-mm straight osteotome. By lifting the bone plaque and periosteum attached to nasal bone (Fig. 4) the cribriform plate was directly exposed, as were both foveae ethmoidale.

The exposed tumor and tumor-invaded ethmoidal bone were
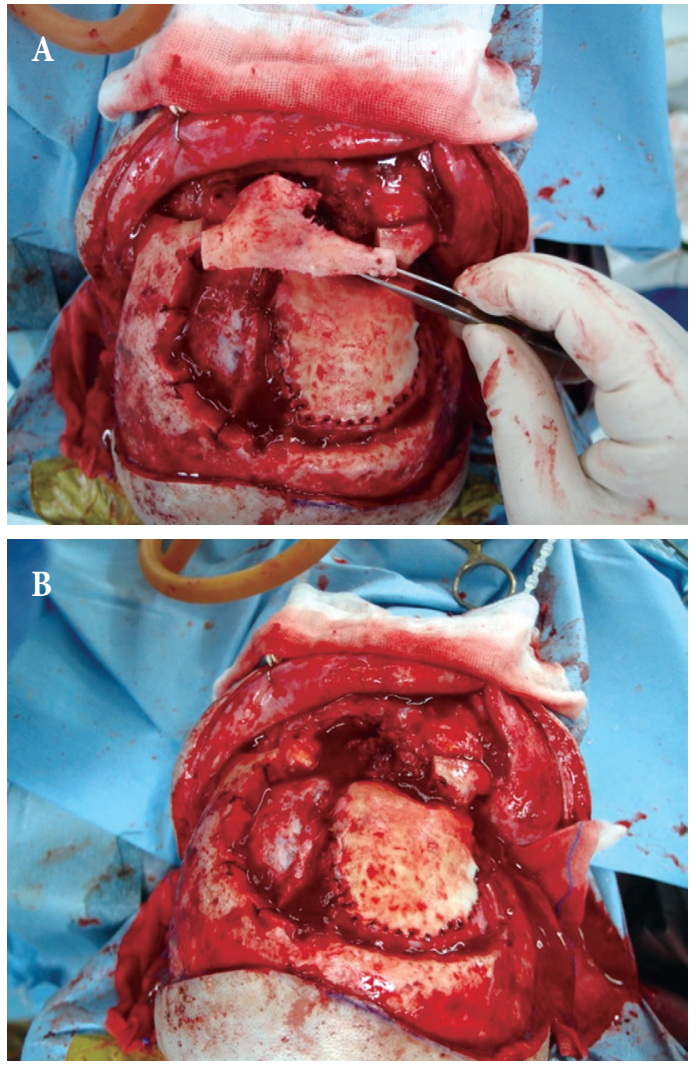

Fig. 4. Intraoperative frontonasal bone segment. (A) The previous dura repair site was exposed via craniotomy. The outline of the frontonasal segment depends on the size and location of the meningioma. Osteotomies are performed across the frontal bone, down to and along the roofs of the orbit, and across the medial wall of the orbit into the nasomaxillary groove just anterior to the lacrimal crest. (B) The frontonasal segment is detached, providing a direct view of the nasal and sphenoethmoidal extracranial parts of the tumor.

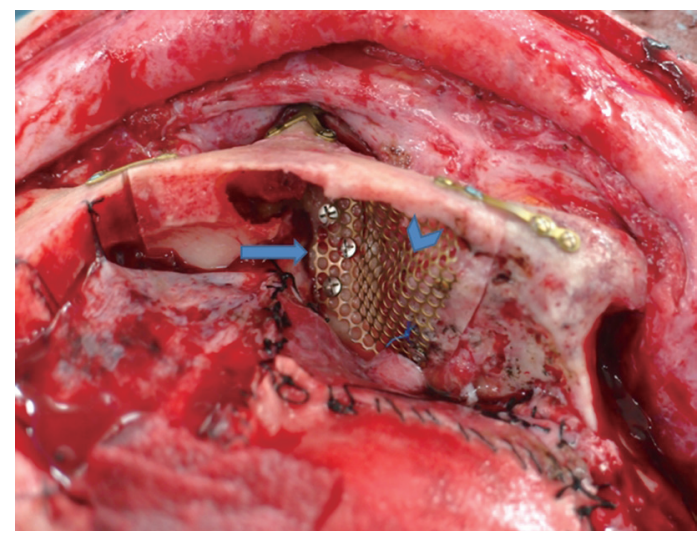

Fig. 5. Intraoperative photograph. The arrowhead indicates the molded titanium mesh. The arrow indicates the calvarial bone graft on resected ethmoidal bone.

resected by the NS. Cranial bone defects were reconstructed by the PS. After removal of the ethmoidal bone, a $2 \times 1 \mathrm{~cm}$ sized calvarial bone was harvested to replace the defect. A titanium mesh was molded for the right orbital roof with tumor invasion by mirroring the left intact orbital roof. A molded titanium mesh was placed at the resection site of the frontal sinus base and anchored to the calvarial bone graft. Repositioning and 3-point fixation of bone plaque was performed with a titanium plate (Fig. 5). The galeal flap was inset to the frontal sinus area. Finally, the frontal flap was closed layer by layer. At postoperative 10 days, all stitches were removed and the patient was discharged. There was no acute complication such as infection, hematoma, or wound dehiscence. During postoperative 6 months there was no subacute complication. The peripheral visual defect (Fig. 2B), EOM limitation, diplopia, and proptosis had disappeared. Postoperative axial CT revealed a $0.46-\mathrm{mm}$ difference between the degrees of the eye proptosis (Fig. 3B). Postoperative visual acuity test scales were 0.8 Log MAR, which were the same as before surgery. Olfactory sense was intact. There was no visible or palpable plate, depression of nose, or cosmetic complication (Fig. 6).

\section{DISCUSSION}

Meningiomas are the most common benign intracranial lesions, representing $18 \%$ of all intracranial neoplasms. Bone is involved in $30 \%$ of these tumors. According to latest data, early and aggres- 

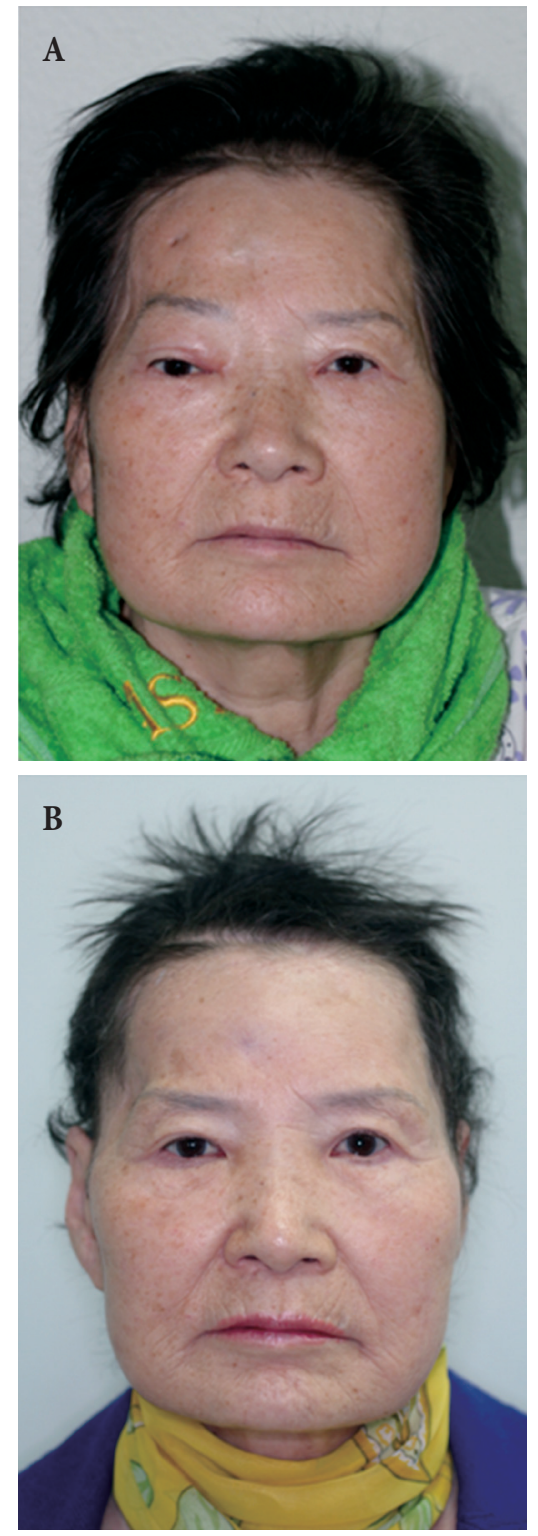

Fig. 6. (A) Preoperative and (B) 6-month postoperative photographs.

sive surgical resection of meningiomas is necessary to prevent recurrences [5]. Hyperostotic lesion of meningioma is associated with tumor invasion, therefore resection of as much hyperostotic bone as possible is important to prevent recurrence of meningioma. However, radical resection increases mortality and morbidity rates and also inevitably leads to postoperative esthetic and functional complications unless suitable reconstruction is performed.

We discussed with the neurosurgery team and decided that we would perform a palliative surgery to conserve part of the supra orbital rim and frontal sinus base in consideration of the patient's age and cosmetic result. There has been no recurrence for 6 months postoperative.

Since the first introduction of the central skull base approach by Raveh et al. in 1988, many approaches have been introduced $[6,7]$. We performed a new modified subcranial approach that allowed direct visualization of the anterior skull tumor with minimal injury of dura and watertight dural reconstruction through broad operative exposure. Most subcranial approaches divide the nasal bone and cartilage joint. However, in this case minimal dissection was performed for tumor resection and separation was performed at the midpoint of the nasal bone leaving 3-5 $\mathrm{mm}$ of distal nasal bone intact to support the upper lateral cartilage. This avoided unnecessary dissection and prevented nasal deviation and depression through titanium fixation between bone and bone. The cosmetic result was also better than with the conventional approach. Conventional transnasal endoscopic procedures are growing in popularity as reliable procedures that avoid skin incisions and minimize surgical morbidity. However, retro-ocular masses and masses far from midline are not accessible transnasally [8]. In this case the tumor mass could not be removed via a transnasal approach.

There are many craniofacial reconstruction materials, such as autogenous bone grafts, chondrocostal grafts, titanium meshes, and other alloplastic materials [9]. Although autologous bone is most widely used it has several limitations such as difficulty in covering large defects, creation of a mold from defect, occurrence of bone resorption, and damage of donor site. In particular, as autologous bone cannot be easily molded it is difficult to match volume symmetry in orbital reconstruction. As a result, enophthalmos develops in 10 to $20 \%$ of patients after surgery [10]. We applied an autologous cavarial bone graft at the ethmoidal portion that does not need much space for molding.

Titanium mesh with an easily controlled shape and volume was applied at the orbital roof. Titanium generally cause less inflammation and conducts well with surrounding mineralized bone [9]. After checking the exact shape and volume of the orbit through imaging such as $\mathrm{CT}$, a precise reconstruction material can be made by 3 -dimensional printing [11]. However, in South 
Korea this is not covered by national health insurance and the cost borne by patients for implants is high. For this reason, we molded titanium mesh from the orbit contour before bone resection. For the damaged side, we molded titanium mesh by mirroring the opposite normal orbit contour. Precise symmetry of volume and shape was achieved using this simple method.

The ideal material for skull reconstruction and surgical approach remains controversial. This case is significant because a single stage without facial skin incision was shown to be safe and effective for removal of gross central lesions. In addition, this approach offers a wide midline exposure of the cranial base and is associated with excellent cosmetic outcomes. However, this procedure is palliative in nature, and therefore it is better to apply it to the elderly and other high-risk patients rather than to all patients.

In conclusion, we successfully performed modified craniofacial osteotomy with partial nasal bone division and removed the tumor. Satisfactory results with regard to esthetics and function were achieved using an autologous bone flap and artificial material.

\section{REFERENCES}

1. Kodera T, Arishima H, Yamada S, Arai H, Akazawa A, Higashino Y, et al. Orbitozygomatic craniotomy with modified zabramski's technique: a technical note and anatomic and clinical findings. World Neurosurg 2017;97:49-57.

2. Kim SR, Lee JW, Han YS, Kim HK. Transfacial surgical approaches to secure wide exposure of the skull base. Arch Craniofac Surg 2015; $16: 17-23$.

3. Feiz-Erfan I, Han PP, Spetzler RF, Porter RW, Klopfenstein JD, Ferreira MA, et al. Exposure of midline cranial base without a facial incision through a combined craniofacial-transfacial procedure. Neurosurgery 2005;56:28-35.

4. Strianese D, Piscopo R, Elefante A, Napoli M, Comune C, Baronissi I, et al. Unilateral proptosis in thyroid eye disease with subsequent contralateral involvement: retrospective follow-up study. BMC Ophthalmol 2013;13:21.

5. Heredero Jung S, Dean Ferrer A, Solivera Vela J, Alamillos Granados F. Spheno-orbital meningioma resection and reconstruction: the role of piezosurgery and premolded titanium mesh. Craniomaxillofac Trauma Reconstr 2011;4:193-200.

6. Raveh J, Laedrach K, Speiser M, Chen J, Vuillemin T, Seiler R, et al. The subcranial approach for fronto-orbital and anteroposterior skullbase tumors. Arch Otolaryngol Head Neck Surg 1993;119:385-93.

7. Kellman RM, Goyal P, Rodziewicz GS. The transglabellar subcranial approach for nasal dermoids with intracranial extension. Laryngoscope 2004;114:1368-72.

8. Wagenmann M, Schipper J. The transnasal approach to the skull base. From sinus surgery to skull base surgery. GMS Curr Top Otorhinolaryngol Head Neck Surg 2011;10:Doc08

9. Cho HR, Roh TS, Shim KW, Kim YO, Lew DH, Yun IS. Skull reconstruction with custom made three-dimensional titanium implant. Arch Craniofac Surg 2015;16:11-6.

10. Schuknecht B, Carls F, Valavanis A, Sailer HF. CT assessment of orbital volume in late post-traumatic enophthalmos. Neuroradiology 1996;38:470-5.

11. Winder J, Cooke RS, Gray J, Fannin T, Fegan T. Medical rapid prototyping and 3D CT in the manufacture of custom made cranial titanium plates. J Med Eng Technol 1999;23:26-8. 